(c) American Dairy Science Association, 2003.

\title{
Sialyloligosaccharides in Human and Bovine Milk and in Infant Formulas: Variations with the Progression of Lactation
}

\author{
S. Martín-Sosa, ${ }^{\star}$ M.-J. Martín, ${ }^{*}$ L.-A. García-Pardo,† and P. Hueso* \\ *Departamento de Bioquímica y Biología Molecular, \\ Facultad de Biología, Universidad de Salamanca, \\ 37007 Salamanca, Spain \\ †Departamento de Fisiología, Facultad de Veterinaria, \\ Universidad de León, \\ 24071 León, Spain
}

\begin{abstract}
Several lines of research support a role for human milk oligosaccharides in the defense of breast-fed infants against pathogens. Some of these oligosaccharides contain at least one moiety of sialic acid and are, thus, termed sialyloligosaccharides. These constitute a significant component ( $>1 \mathrm{~g} / \mathrm{L}$ ) of human milk. It is well established that milk composition varies among species, and previous reports have indicated that one of the differences between human and bovine milk is precisely their contents of sialyloligosaccharides. Because most infant formulas are manufactured with bovine milk components, it follows that formula-fed and breast-fed infants ingest dissimilar quantities of these carbohydrate structures. To ascertain these differences and their impact along lactation, the contents of oligosaccharide-bound sialic acids and major sialyloligosaccharides in samples of human and bovine milk (obtained at different lactation stages) were determined. In addition, infant formulas were assayed for their sialyloligosaccharide contents. Seven sialyloligosaccharides were identified in human milk; namely, $3^{\prime}$-sialyl-3-fucosyllactose and sialyllacto- $N$-tetraoses (a and $b+c)$, the predominant structures at all lactation stages. Five sialyloligosaccharides were identified in bovine milk, of which 6 '-sialyllactosamine and $3{ }^{\prime}$-sialyllactose were the most abundant. In addition, sialyloligosaccharides in human and bovine milk decreased along lactation, and infant formulas did not contain significant amounts of sialyloligosaccharides. The results point to the general conclusion that regarding both qualitative and quantitative aspects, milk from humans and cows and infant formulas have different oligosaccharide contents. In this sense, bottle-fed infants are subject to reduced sialyloligosaccharide intake as compared to breast-fed infants.
\end{abstract}

Received June 14, 2002.

Accepted August 5, 2002.

Corresponding author: P. Hueso; e-mail: phueso@usal.es.
(Key words: Infant formula, human/bovine lactation, milk oligosaccharide)

Abbreviation key: DSL = disialyllactose, DSLNT = disialyllacto- $N$-tetraose, LSTa $=$ sialyllacto- $N$-tetraose a, LSTb = sialyllacto- $N$-tetraose b, LSTc $=$ sialyllacto$N$-tetraose c, OBSA = oligosaccharide-bound sialic acid, $\mathbf{R T}=$ retention time, $\mathbf{3}^{\prime} \mathbf{S} \mathbf{} \mathbf{F L}=3^{\prime}$-sialyl-3-fucosyllactose, $\mathbf{3}^{\prime} \mathbf{S L}=3^{\prime}$-sialyllactose, $\mathbf{3}^{\prime} \mathbf{S L N}=3^{\prime}$-sialyllactosamine, $6^{\prime} \mathbf{S L}=6^{\prime}$-sialyllactose, $\mathbf{6}^{\prime} \mathbf{S L N}=6^{\prime}$-sialyllactosamine.

\section{INTRODUCTION}

Oligosaccharides are an important constituent of milk (the third solid component after lactose and fat in human milk). Human milk has a high content of oligosaccharides, not only in colostrum (20 to $23 \mathrm{~g} / \mathrm{L}$ ) but also in mature milk (12 to $14 \mathrm{~g} / \mathrm{L}$; Coppa et al., 1999). Cow's milk has a content in oligosaccharides that is about 20 -fold lower than that found in human milk ( 0.7 to $1.2 \mathrm{~g} / \mathrm{L}$ in colostrum; Veh et al., 1981). More than 130 complex oligosaccharides have been isolated and characterized in human milk (McVeagh and Miller, 1997), but little information is available about these compounds in bovine milk. Recently, a review by Gopal and Gill (2000) reported 10 sialylated and eight neutral oligosaccharides in bovine milk and colostrum. Human milk is considered to be unique in its high content of complex fucosylated and sialylated oligosaccharides (Kunz et al., 1999). Conversely, bovine milk contains mainly sialylated species (Veh et al., 1981; Saito et al., 1984).

It has been described that certain oligosaccharides inhibit specific pathogen-binding to host cell receptors in the intestinal tract. Fucosyloligosaccharides from human milk inhibit the diarrheagenic effect of the heatstable toxin of Escherichia coli and Campylobacter jejuni. The binding of Streptococcus pneumoniae, enteropathogenic E. coli and Haemophilus influenza to their receptors is also inhibited by human milk oligosaccharides (for a review, see Kunz and Rudloff, 1993; 
McVeagh and Miller, 1997; Newburg, 1997, 1999). Other oligosaccharides would protect against other pathogens. In in vitro assays, human milk oligosaccharides are minimally digested (Engfer et al., 2000; Gnoth et al., 2000), a prerequisite for them to be effective in the small intestine in preventing infections. However, little is known about the digestion of milk oligosaccharides during their passage through the calf gastrointestinal tract.

The milk contents of each species are specifically tailored to meet the special needs of the newborns. Therefore, the aim of this work was to carry out a comparative study of the oligosaccharide contents of human and bovine milk from different stages of lactation. Sialyloligosaccharides were chosen since they are the most abundant oligosaccharides in bovine milk (about 80\%) and are found in high amounts in human milk (about $1 \mathrm{~g} / \mathrm{L})$. Breast-fed infants appear to have fewer or less severe gastrointestinal, respiratory and urinary infections than formula-fed infants (Kovar et al., 1984; Marild et al., 1990). Infant formula manufacturers have tried to emulate human milk in macronutrient composition and occasionally with respect to the actual molecular components. In addition, various substances, including oligosaccharides, have been attributed functional effects in certain special situations. Because the infant formulas designed for nutrition during the first weeks after birth are currently produced from different bovine milk derivatives, their sialyloligosaccharide contents were also compared to those of bovine and human milks.

\section{MATERIALS AND METHODS}

\section{Milk Samples}

Human milk was collected with a breast pump from 12 healthy volunteer Spanish donors who had delivered at term. Each woman donated three samples from three different lactation stages. Colostrum (d 1 to 4 ), transitional (d 12 to 17 ) and mature milk (d 28 to 32 ) samples were collected, immediately frozen at $-20^{\circ} \mathrm{C}$, lyophilized, and homogenized to ensure accurate distribution of the components. Donors were primiparous/multiparous women between the ages of 25 and 35 .

Samples from fresh cow milk and colostrum were obtained from six individual multiparous SpanishBrown cows at Huergas de Babia (León). Each cow provided four samples from four different lactation stages: Colostrum (d 2), transitional milk (d 7), mature milk (mo 3), and late lactation milk (mo 10) samples were obtained from the morning milking, immediately frozen at $-20^{\circ} \mathrm{C}$, lyophilized, and homogenized.

Five infant formulas (chosen from those most frequently consumed in Spain) from three different baby food companies were analyzed. These are recommended for use from birth to 3 to 4 mo of age. All formulas are bovine-milk based.

\section{Oligosaccharide Standards}

Several sialyloligosaccharide standards from human milk and bovine colostrum of known concentration were injected to obtain calibration curves on an HPLC apparatus. Disialyllacto- $N$-tetraose (DSLNT) 3 '-sialyllactose (3'SL), 6'-sialyllactose (6'SL), 3'-sialyllactosamine (3'SLN), 6'-sialyllactosamine (6'SLN), 3'-sialyl-3-fucosyllactose (3'S3FL), sialyllacto- $N$-tetraose a (LSTa), sialyllacto- $N$-tetraose b (LSTb), and sialyllacto- $N$-tetraose c (LSTc) were purchased from Glyko (Upper Heyford, UK). Disialyllactose (DSL) was purchased from Sigma (St. Louis, MO).

\section{Oligosaccharide-Bound Sialic Acid Quantitation}

For quantitation of oligosaccharide-bound sialic acid (OBSA) contents, lyophilized milk samples and formulas were reconstituted in water according to their original water content or the manufacturers' instructions, respectively; defatted by centrifugation $(3000 \times g, 30$ min at $4^{\circ} \mathrm{C}$ ), and filtered through glass wool. The filtrates were mixed with an equal volume of a $24 \%$ TCA (Sigma) solution, allowed to precipitate for $10 \mathrm{~min}$ on ice after vortexing, and centrifuged at $3000 \times g$ for 30 min at $4^{\circ} \mathrm{C}$. Protein precipitates were washed twice with cold $12 \%$ TCA to remove any traces of carbohydrates. After this, the supernatant solution and washings were combined and hydrolyzed in $0.05 \mathrm{M} \mathrm{H}_{2} \mathrm{SO}_{4}$ at $80^{\circ} \mathrm{C}$ for $1 \mathrm{~h}$. The hydrolysates were cooled to room temperature and purified by ion-exchange chromatography on a Dowex $2 \times 8$ column $(2 \mathrm{ml})$, formic form. Sialic acids were eluted with $0.1 M$ formic acid, lyophilized, dissolved in distilled water, and used to determine OBSA contents (Martín et al., 2001). They were then quantitated by the resorcinol procedure of Svennerholm (1957). Assays were made in triplicate; i.e., each individual sample was analyzed three times.

\section{Isolation of the Oligosaccharide Fraction}

Isolation of total oligosaccharides was performed as described by Kobata (1972), with several modifications. Briefly, samples were defatted by centrifugation (3000 $\times g, 30 \mathrm{~min}, 4^{\circ} \mathrm{C}$ ) and filtered through glass wool. The filtrates were mixed with two volumes of ethanol and allowed to stand overnight at $4^{\circ} \mathrm{C}$. Most proteins and lactose precipitated and were removed by centrifugation at $0^{\circ} \mathrm{C}$ and evaporating off the ethanol. The crude oligosaccharide fraction was further purified by molecular exclusion chromatography (Sephadex G-25) to elimi- 
nate residual proteins and peptides. Ninhydrin $(0.3 \mathrm{~g}$ of ninhydrin in $95 \mathrm{ml}$ of butanol) -positive fractions were discarded. Orcinol $\left(0.1 \%\right.$ orcinol in $\left.20 \% \mathrm{H}_{2} \mathrm{SO}_{4}\right)$ positive fractions were collected together and kept at $-20^{\circ} \mathrm{C}$ until use.

\section{HPLC Analysis}

Sialylated oligosaccharide contents were analyzed by HPLC according to the method previously described by Michalski (1995). Analyses were performed on a Waters apparatus (Waters, Milford, MA), using an $\mathrm{NH}_{2}$-bound silica column (Carbohydrate Analysis, $300 \times 3.9 \mathrm{~mm}$, Waters). HPLC elution of bovine milk samples was performed with a gradient of acetonitrile and $15 \mathrm{mM}$ $\mathrm{KH}_{2} \mathrm{PO}_{4}$ adjusted to $\mathrm{pH}$ 5.2: isocratic elution with $25 \%$ $15 \mathrm{mM} \mathrm{KH} \mathrm{KO}_{4}$ for $15 \mathrm{~min}$, linear gradient up to $50 \%$

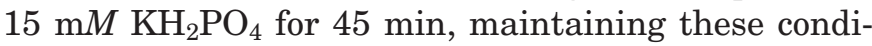
tions for $15 \mathrm{~min}$. Flow rate was $1 \mathrm{ml} / \mathrm{min}$. Samples of human milk and infant formulas were eluted under almost identical conditions, except that isocratic elution at $25 \% \mathrm{KH}_{2} \mathrm{PO}_{4}$ lasted $10 \mathrm{~min}$, and the linear gradient to $50 \%$ lasted $50 \mathrm{~min}$ for better separation. Oligosaccharides were detected at $206 \mathrm{~nm}$ and were identified by comparison of their retention times (RT) with those of standard oligosaccharides injected previously. Oligosaccharide quantification was carried out from the calibration curves of standard oligosaccharides using Millenium software (Waters) coupled to the HPLC system. All assays were carried out in triplicate.

\section{Neuraminidase Treatment}

Neuraminidase (E.C.3.2.1.18) from Clostridium perfringens was purchased from Sigma. Milk oligosaccharide samples were treated with neuraminidase in 50 $\mathrm{m} M$ sodium acetate buffer, $\mathrm{pH} 5.5$, for $16 \mathrm{~h}$ at $37^{\circ} \mathrm{C}$. Digested oligosaccharides were later analyzed by HPLC.

\section{Statistical Analysis}

Results are expressed as means \pm SD. Oligosaccharide differences among stages were tested by one-way ANOVA using the SPSS 9.0.1 program for Windows (SPSS Inc., Chicago, IL). The Scheffé test was used for comparisons between groups. Differences were considered significant at a probability level of $P<0.01$.

\section{RESULTS}

\section{OBSA Quantitation}

The amount of sialic acids bound to the oligosaccharide fraction was determined in all samples. As shown in Table 1, the OBSA content decreased along lactation, colostrum being the significantly different stage. In bovine milk, the slight increase in OBSA contents in the late lactation stage was not statistically significant. Infant formulas proved to be quite heterogeneous in their OBSA content (14.6 to $68.6 \mathrm{mg} / \mathrm{L})$. In all cases, the average value obtained $(38.1 \mathrm{mg} / \mathrm{L})$ was quite low with respect to human milk and similar to that of midlactation bovine milk.

\section{HPLC Identification of Sialylated Oligosaccharides}

To identify the individual sialyloligosaccharides, total oligosaccharides from each stage were injected in the HPLC apparatus. Under the described conditions, only acidic compounds were resolved and detected.

Human milk. Along human lactation, a very similar chromatographic profile was observed (Figures 1a and 1b) from one stage to another. The chromatograms displayed 11 major peaks, but only seven of them could be identified using the standards available. These peaks corresponded to the following oligosaccharides: 3 'SL, 6'SL, 3'S3FL, LSTa (NeuAc $\alpha 2-3 \mathrm{Gal} \beta 1-3$ GlcNAc $\beta 1$-3 Gal $\beta 1-4$ Glc), LSTb [Gal $\beta 1-3$ (NeuAc $\alpha 2-6$ ) GlcNAc $\beta 1$ 3 Gal $\beta 1-4$ Glc], LSTc (NeuAc $\alpha 2-6$ Gal $\beta 1-4$ GlcNAc $\beta 1$ 3 Gal $\beta 1-4$ Glc), and DSLNT. LSTb and LSTc had very similar RT, and were, therefore, considered as LSTb+c, although it was not possible to know what percentage of the area corresponded to each of them. $3^{\prime} \mathrm{SLN}, 6^{\prime} \mathrm{SLN}$, and DSL were not found in any of the human samples. The first peak in the chromatogram (RT:12.5 min) appeared slightly later than the large neutral oligosaccharide peak (not shown). The material in this peak was isolated and proved to be ninhydrin-positive, orcinolpositive, and resorcinol-negative. Most of the acidic oligosaccharides identified decreased significantly along lactation (Figure 2). 3'SL did not vary significantly along lactation. The most representative constituents were 3'S3FL, LSTa, and LSTb+c from among all the sialyloligosaccharides identified in this study. Peaks with an RT of $>45$ min could not be identified. The sialyloligosaccharide composition of individual women varied mainly with respect to one of the component, i.e., 3 '-sialyl-3-fucosyllactose. Three of the women had high amounts of this sialyloligosaccharide (Figure 1), while the rest of the women had more moderate amounts.

Bovine milk. As in human milk, the chromatographic oligosaccharide profile was quite homogeneous along lactation, although there were significant differences among the different stages. Five peaks were identified and were present at each stage. Between min 15 and 25 (RT), four sialylated trisaccharides were clearly identified as 3'SLN, 3'SL, 6'SLN, and 6'SL; 3'S3FL was 
Table 1. Oligosaccharide-bound sialic acids in milk and infant formulas. ${ }^{1}$

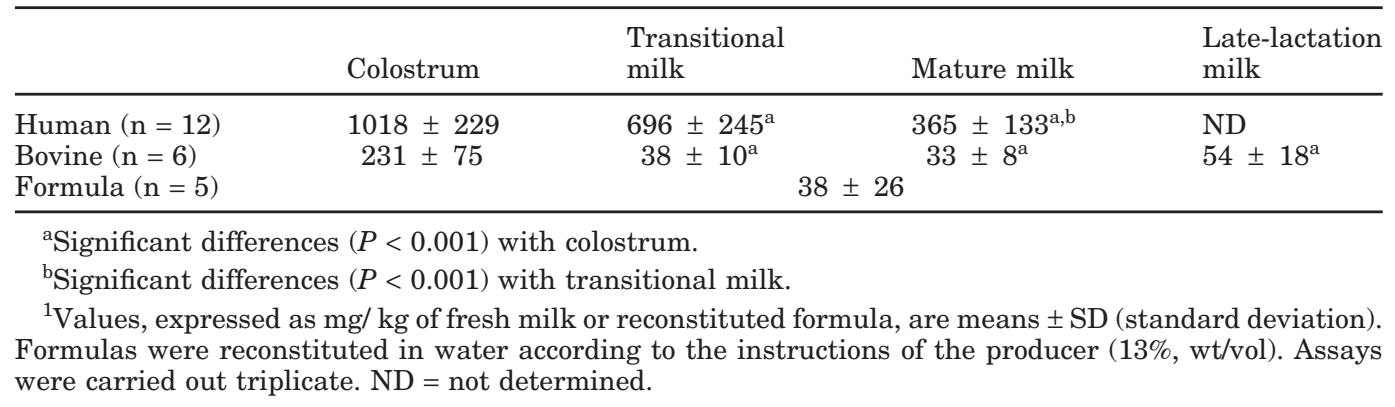

not present at any of the stages. The only disialyloligosaccharide identified was DSL; DSLNT was not found. In some chromatograms, small peaks with $\mathrm{RT}$ values similar to those of the sialylated tetraose standards appeared. Neither the first peak of the chromatograms (named peak "a"), nor peaks with an RT $>45$ min were identified. The material corresponding to peak "a" was isolated and proved to be ninhydrin-positive, orcinolpositive, and resorcinol-negative. Figure 3 shows the quantitative variations along lactation. Most of the significant differences were seen with respect to the colostrum, which was the stage with the highest values in most of the oligosaccharides identified. Nevertheless, 3'SLN was significantly higher in transitional milk.

Infant formulas. The chromatographic profiles obtained from infant formulas (Figure 1d) were generally characterized by the absence of peaks. Only one unidentified peak was present in all the chromatograms. This peak had the same RT as peak "a" in bovine milk. In some chromatograms, there were trace peaks of 3 'SL, 6'SL, or unidentified compounds.

\section{Neuraminidase Treatment}

When oligosaccharides from human milk were treated with neuraminidase, all the peaks disappeared from the chromatographic profile; only those with an $\mathrm{RT}<8$ min (neutral oligosaccharides) remained. The peak with an RT of 12.5 min did not disappear. After enzymatic treatment of bovine samples, only peak "a" remained unaltered in the chromatograms; the rest of the peaks disappeared.

\section{DISCUSSION}

The decreasing trend along lactation in the OBSA content observed in the present study, not only in human but also in bovine milk, seems clear. Nevertheless, the data in the literature concerning bovine milk OBSA vary widely. Regarding both colostrum and mature milk, the data obtained in our research lie at an intermediate level between those previously reported (Veh et al., 1981; Parkkinen and Finne, 1987). Concerning human milk OBSA, our data are in agreement with what has been previously reported (Carlson, 1985). The OBSA content of infant formulas varied widely from one to another. This variation could be due to differences in the casein:whey ratio. Formulas with a high proportion of whey are probably richer in soluble oligosaccharides (which were already present in cow's milk) than those with a higher casein content. However, this information was not given for all the formulas studied and, hence, no definitive conclusions can be drawn. The data in the literature also reflect this heterogeneity, the published values ranging from 14 to $174 \mathrm{mg} / \mathrm{kg}$ (Carlson, 1985; Neeser et al., 1991; Sánchez-Díaz et al., 1997). In any case, the OBSA content of infant formulas is more similar to that of bovine milk than that of human milk.

Because treatment of human milk oligosaccharide samples with neuraminidase yielded a chromatogram lacking peaks (except for the peak with an RT of 12.5 min, which was assumed to be a retarded neutral oligosaccharide), all of the peaks in Figure $1 \mathrm{a}$ and $1 \mathrm{~b}$ can be said to contain a sialic acid residue. No important qualitative changes in the sialyloligosaccharide chromatographic profile were observed from one stage to another. Nevertheless, a significant quantitative difference was observed between colostrum and mature milk, consistent with the overall tendency for OBSA to decrease. Considering the sialyloligosaccharides individually, in general, we obtained slightly higher values than those previously reported (Kunz and Rudloff, 1993; Coppa et al., 1999). Nonetheless, the significant differences among the stages reported in previous works were essentially in agreement with our own findings.

The data reported in the literature (Coppa et al., 1999) refer to a fairly constant value along lactation for LSTb, while LSTc apparently declines sharply in the mature milk. This prompts us to speculate that the significant decrease in our LSTb+c peak would be mainly due to the LSTc fraction.

A major sialyloligosaccharide species in human milk, DSLNT, has not previously been reported to decrease along lactation. Although we found the same amount 


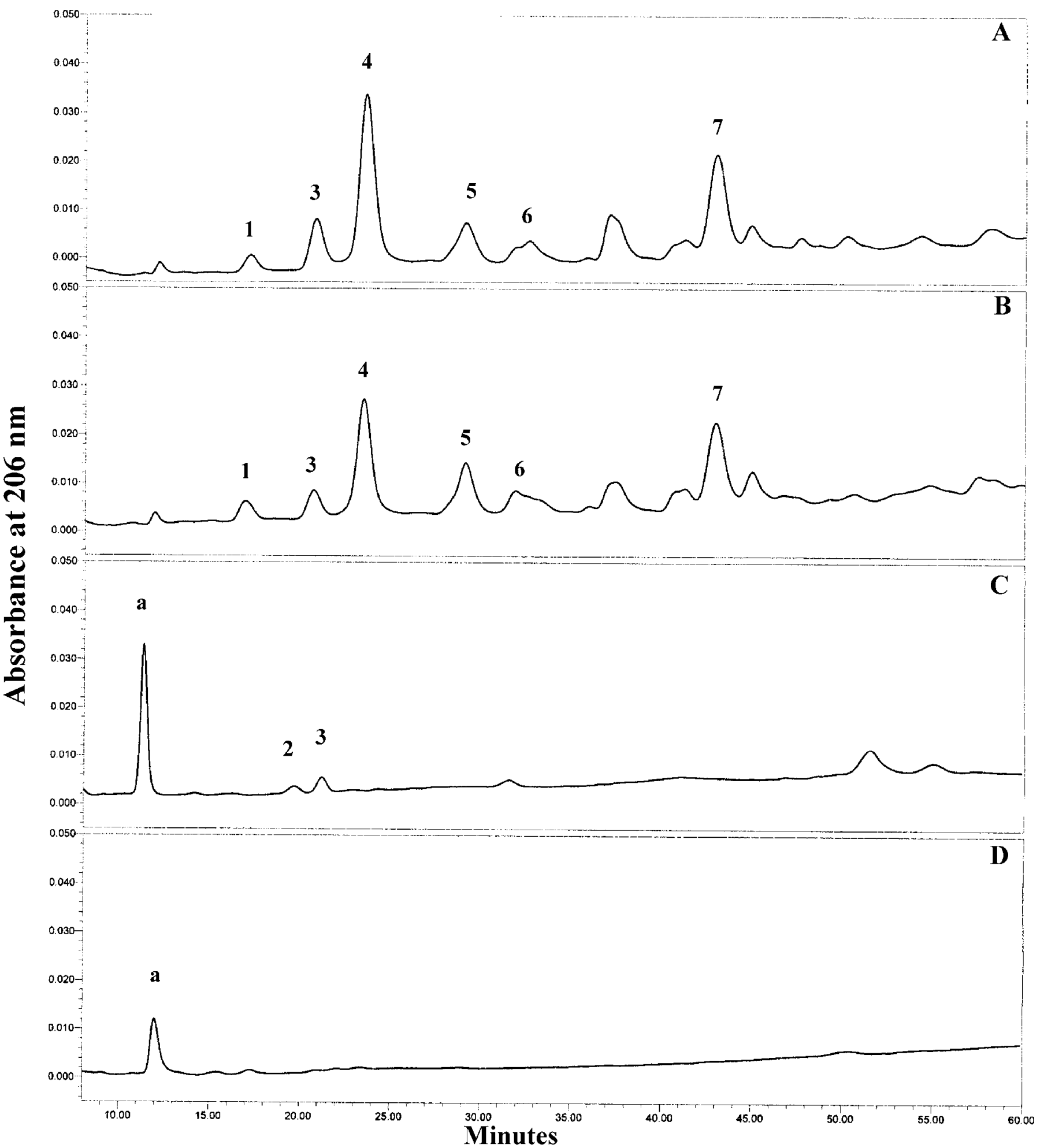

Figure 1. HPLC profiles of sialyloligosaccharides. A, human colostrum; B, human mature milk; C, bovine mature milk; D, infant formula. Peak 1: 3'sialyllactose; 2:6'sialyllactosamine; 3: 6'sialyllactose; 4: 3'sialyl-3fucosyllactose; 5: sialyllacto- $N$-tetraose a; 6: sialyllacto- $N$-tetraose b + sialyllacto- $N$-tetraose c; 7: disialyllacto- $N$-tetraose. Peak “a”: unidentified resorcinol-negative peak. 


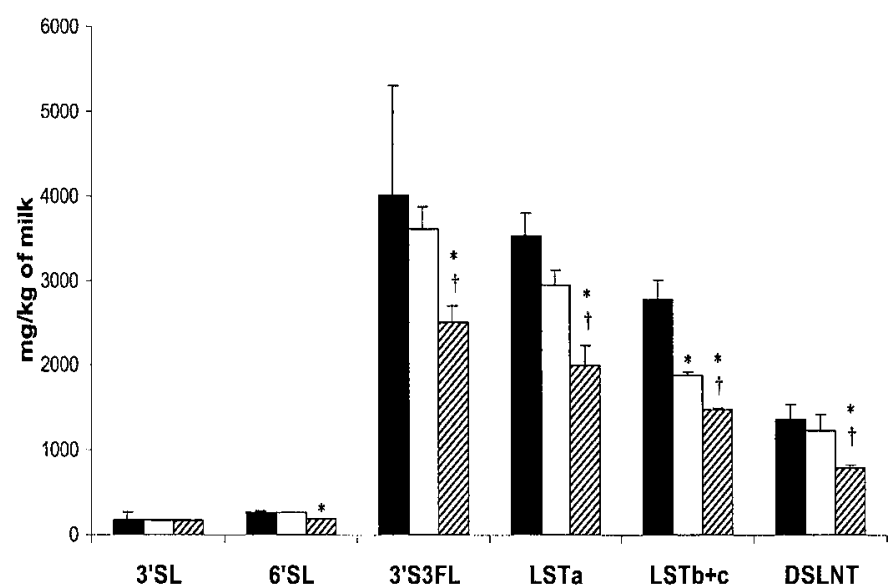

Figure 2. Distribution of the different sialyloligosaccharides identified in human milk along lactation. Colostrum: black bars, Transition milk: white bars, Mature milk: hatched bars. 3'SL; 3'-sialyllactose, 6'SL; 6'-sialyllactose, 3'S3FL; 3'-sialyl-3-fucosyllactose, LSTa; sialyllacto- $N$-tetraose a, LSTb + c; sialyllacto- $N$-tetraose b + sialyllacto- $N$-tetraose c, DSLNT; disialyllacto- $N$-tetraose. *: Significant differences $(P<0.001)$ with colostrum. $\dagger$ : Significant differences $(P<$ $0.001)$ with transitional milk. Values are means $\pm \mathrm{SD}(\mathrm{n}=12)$.

of DSLNT in our mature milk samples as in previous works (Kunz and Rudloff, 1993; Coppa et al., 1999), our much higher values for colostrum could explain the significant descending trend observed by us.

We found large amounts of 3'S3FL in all three stages of lactation of three of the donors. Although its presence in human milk has been described previously (Gröndberg et al., 1989), it has not been reported to be an important sialylated oligosaccharide in other quantitative studies (Kunz and Rudloff, 1993; Coppa et al., 1999). We are unaware of whether the presence of 3'S3FL in our samples is a genetic-dependent phenomenon. Human milk samples were collected from private donors, and no information about their blood groups was available.

Studies on individual variations in milk samples have been published (Newburg and Neubauer, 1995; Kunz et al., 1999). The relative proportions of $3^{\prime} \mathrm{SL}, 6^{\prime} \mathrm{SL}$, and sialyltetraoses have been found to vary among individuals. Because diurnal differences in oligosaccharide contents have also been reported (Viverge et al., 1986), the level of variation seems to be extremely high. Nonetheless, the biological relevance of such variations remains obscure.

Parallel experiments carried out in our laboratory with human milk from Cuban donors showed a very similar composition in the sialyloligosaccharide content. However, we found a statistically significant difference in the LSTb+c fraction (data not shown). While in colostrum, Cuban women seemed to have lower amounts of LSTb+c than Spanish mothers, in transi-

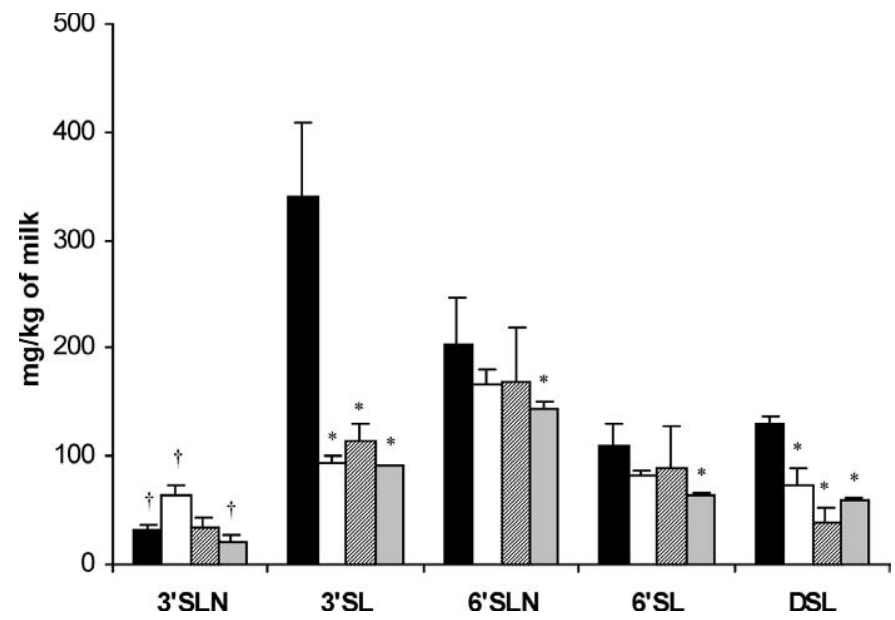

Figure 3. Distribution of the different sialyloligosaccharides identified in bovine milk along lactation. Colostrum: black bars, Transition milk: white bars, Mature milk: hatched bars, Late-lactation milk: shaded bars. 3'SLN; 3'-sialyllactosamine, 3'SL; 3'-sialyllactose, 6'SLN; 6'-sialyllactosamine, 6'SL; 6'-sialyllactose, DSL; disialyllactose.*: Significant differences $(P<0.001)$ with colostrum. $\dagger$ : Significant differences $(P<0.001)$ with transitional milk. Values are means \pm $\mathrm{SD}(\mathrm{n}=6)$

tional milk, the proportions of these tetraoses switched. Variations among different populations have been reported previously (Erney et al., 2000), but further experiments in this field need to be done.

Bovine milk contains acidic rather than neutral oligosaccharides (Saito et al.,1984). To date, 10 sialylated oligosaccharides have been described (Gopal and Gill, 2000). The main species identified in our work were 3'SL, 6'SLN, 6'SL, and DSL, which in bovine colostrum have already been reported to be predominant (Newburg et al., 1996). No data about sialylated oligosaccharides at other stages of lactation have been found.

We found a small amount of 3'SLN in the four stages considered, transitional milk being the richest in this compound. This species has not been previously reported in bovine colostrum or milk.

As in previous reports (Veh et al., 1981; Parkkinen and Finne, 1987), we found 3'SL to be the most representative species in bovine colostrum, although $6^{\prime}$ SLN remained at constantly high values along lactation. Regarding the rest of the species identified, except for DSL, our values are appreciably higher than those reported in the literature. The presence of $\alpha 2-3$ isomers diminished along bovine lactation.

For all the sialyloligosaccharides identified, except 3'SLN, a significant difference was seen between the colostrum and other lactation stages. From a nutritional point of view, we consider the sialyloligosaccharide composition of mature stages to be very interesting, 
as it is commonly used in the manufacture of infant formulas.

In some chromatograms, we found trace peaks with $\mathrm{RT}$ values similar to those of the sialyltetraose standards. Sialyltetraoses have not been previously reported in cow's milk or colostrum, so we cannot assign any structure without further studies. Urashima and coworkers (1991) described a neutral lacto- $N$-neo-tetraose. It is clear that individual breeds, diet, the length of the dry period of cows, and time postparturition make it difficult to assume a typical composition profile (Gopal and Gill, 2000).

With respect to the first peak, "a", in the bovine milk chromatograms, this was assumed to be a retarded neutral oligosaccharide, since it mostly remained unaltered after digestion with neuraminidase.

As shown in Figure 1d, the chromatographic profile of infant formulas was different from that of bovine milk or human milk. Nevertheless, the chromatograms of the individual infant formulas differed from one to another, as some of them were totally flat (total absence of peaks). By contrast, others had very low amounts of 3'SL and 6'SL or showed unidentified trace peaks. In any case, they resembled those from bovine mature milk more than those from human colostrum and milk.

Since several studies have reported that most human milk oligosaccharides are not digested nor absorbed into the small intestine of infants (Engfer et al., 2000; Gnoth et al., 2000), it is reasonable to speculate that they could play a defensive role, among others. In this sense, several reports about the inhibition of microbial adhesion to host cells by fucosylated and sialylated oligosaccharides have been published (Kunz and Rudloff, 1993; McVeagh and Miller, 1997; Newburg, 1997, 1999). In calves, sialylated oligosaccharides could play the same role before they become ruminants. Indeed, experiments carried out at our laboratory have demonstrated that bovine milk sialylated oligosaccharides bind to several enterotoxigenic $E$. coli strains isolated from calves (unpublished data).

The bioactivity of oligosaccharides from bovine and human milk would be similar and, therefore, bovine oligosaccharides could be used in milk products as bioactive components in human nutrition (Gopal et al., 2000). Nonetheless, breast-fed infants are better protected against several types of infections than formulafed infants (Newburg, 1997). Infant formulas are designed specifically to mimic the composition of human milk or the functional aspects of human breast-feeding. The idea of supplementing formula milk with oligosaccharides similar to those found in human milk has been discussed previously (McVeagh and Miller, 1997; Motil, 2000). Large-scale production of oligosaccharides is currently being investigated, and its application in infant nutrition has been suggested (Endo and Koizumi, 2000). However, further research is needed as regards the structural identity, form, amounts, stability, and biological role of human milk oligosaccharides before this step can be taken.

\section{CONCLUSIONS}

The OBSA content of bovine milk is between 4.4 and 18 times lower than that of human milk, depending on the stage of lactation. Infant formulas have an OBSA content similar to that of transitional or mature bovine milk and consequently lower than that of human milk (between 10 and 27 times). Seven oligosaccharides in human milk and five in bovine milk have been identified in several stages of lactation, ranging from colostrum to late lactation milk. The most abundant are 3 'S3FL and LSTa in human milk, and 6'SLN and 3'SL in bovine milk. A marked heterogeneity in the oligosaccharide content, mainly that of 3 'S3FL, was found among women. Infant formulas only had trace peaks of 3'SL, 6'SL, or unidentified compounds.

The results point to the general conclusion that, regarding both qualitative and quantitative aspects, milk from humans and cows and infant formulas have different oligosaccharide contents. The manufacturing process for infant formulas has virtually eliminated OBSA. In this sense, bottle-fed infants are subject to reduced sialyloligosaccharide intake as compared to breastfed infants.

\section{ACKNOWLEDGMENTS}

This work was partly supported by grants from the Programa de Apoyo a Proyectos de Investigación de la Junta de Castilla y León, España (SA 68/98) and the Ministerio de Educación y Cultura de España (CICYT; ALI 95- 0532).

We acknowledge the generous collaboration of the private donors, as well as the great help given by Mrs. Esmeralda Gómez de Miguel, midwife from the Health Center "La Alamedilla" (Salamanca, Spain), who kindly provided us with a large part of the samples. We thank Mr. Gerardo Álvarez, who carefully collected all the bovine samples. We also wish to thank Dr. Gilda Marquina from the Center of Molecular Immunology (Havana, Cuba) for providing the Cuban samples. We are also grateful to Mr. N. Skinner (from the Servicio de Idiomas, Universidad de Salamanca) for revising the English version of the manuscript.

\section{REFERENCES}

Carlson, S. E. 1985. $N$-acetylneuraminic acid concentrations in human milk oligosaccharides and glycoproteins during lactation. Am. J. Clin. Nutr. 41: 720-726. 
Coppa, G. V., P. Pierani, L. Zampini, I. Carloni, A. Carlucci, and O. Gabrielli. 1999. Oligosaccharides in human milk during different phases of lactation. Acta Pediatr. 430 suppl:89-94.

Emmett, P. M., and I. S. Rogers. 1997. Properties of human milk and their relationship with maternal nutrition. Early Hum. Dev. 49 Suppl :S7-S28

Endo, T., and S. Koizumi. 2000. Large-scale production of oligosaccharides using engineered bacteria. Curr. Opin. Struc. Biol. 10:536-541.

Engfer, M. B., B. Stahl, G. Sawatzki, and H. Daniel. 2000. Human milk oligosaccharides are resistant to enzymatic hydrolysis in the upper gastrointestinal tract. Am. J. Clin. Nutr. 71: 1589-1596.

Erney, R. M., W. T. Malone, M. B. Skelding, M. B. Marcon, K. M. Kleman-Leyer, M. L. O’Ryan, G. Ruiz-Palacios, M. D. Hilty, L. K. Pickering, and P. A. Prieto. 2000. Variability of human milk neutral oligosaccharides in a diverse population. J. Pediatr. Gastroenterol. Nutr. 30:181-192.

Finke, B., B. Stahl, A. Pfenninger, M. Karas, H. Daniel, and G. Sawatzki. 1999. Analysis of high-molecular-weight oligosaccharides from human milk by liquid chromatography and MALDI-MS. Anal. Chem. 71:3755-3762.

Gnoth, M. J., C. Kunz, E. Kinne-Saffran, and S. Rudloff. 2000. Human milk oligosaccharides are minimally digested in vitro. J. Nutr. 130:3014-3020.

Gopal, P. K., and H. S. Gill. 2000. Oligosaccharides and glycoconjugates in bovine milk and colostrum. Br. J. Nutr. 84:569-574.

Gröndberg, G., P. Lipniunas, T. Lundgren, K. Erlansson, F. Lindh, and B. Nilsson. 1989. Isolation of monosialylated oligosaccharides from human milk and structural analysis of three new compounds. Carbohydr. Res. 191:261-278.

Guerardel, Y., W. Morelle, Y. Plancke, J. Lemoine, and G. Strecker. 1999. Structural analysis of three sulphated oligosaccharides isolated from human milk. Carbohydr. Res. 320:230-238.

Kobata, A. 1972. Isolation of oligosaccharides from human milk. Methods Enzymol. 28:262-271.

Kovar, M. G., M. K. Sedula, J. S. Marks, and D. W. Frase. 1984. Review of the epidemiologic evidence for an association between infant feeding and infant health. Pediatrics 74:615-638.

Kunz, C., and S. Rudloff. 1993. Biological functions of oligosaccharides in human milk. Acta Pediatr. 82:903-912.

Kunz, C., S. Rudloff, W. Schad, and D. Braun. 1999. Lactose-derived oligosaccharides in the milk of elephants: comparison with human milk. Br. J. Nutr. 82:391-399.

McVeagh, P., and J. Brand Miller. 1997. Human milk oligosaccharides: only the breast. J. Paediatr. Child H. 33:281-286

Marild, S., U. Jodal, and L. A. Hanson. 1990. Breast-feeding and urinary tract infection. Lancet 336:942
Martín, M. J., S. Martín-Sosa, L. A. García-Pardo, and P. Hueso. 2001. Distribution of bovine milk sialoglycoconjugates during lactation. J. Dairy Sci. 84:995-1000.

Michalski, J. C. 1995 . Isolation of glycans by HPLC. Pages $67-77$ in Methods on glycoconjugates. A.Verbert, ed. Harwood Academic Publishers, Churr, Switzerland.

Motil, K. J. 2000. Infant feeding: a critical look at infant formulas. Curr. Opin. Pediatr. 12:469-476.

Neeser, J. R., M. Golliard, and S. Del Vedovo. 1991. Quantitative determination of complex carbohydrates in bovine milk and in milk-based infant formulas. J. Dairy Sci. 74:2860-2871.

Newburg, D. S., and S. H. Neubauer. 1995. Carbohydrates in milks: analysis, quantities, and significance. Pages 273-349 in Handbook of milk composition. R. G. Jensen, ed. Academic Press, San Diego, CA

Newburg, D. S., P. Chaturvedi, C. D. Warren, S. Lui, G. M. RuizPalacios, and L. K. Pickering. 1996. Variation of human milk oligosaccharide profiles in a normal population. Pediatr. Res. 39:229A.

Newburg, D. S. 1997. Do the binding properties of oligosaccharides in milk protects human infants from gastrointestinal bacteria? J. Nutr. 127:980S-984S.

Newburg, D. S. 1999. Human milk glycoconjugates that inhibit pathogens. Curr. Med. Chem. 6:117-127

Parkkinen, J., and J. Finne. 1987. Isolation of sialyl oligosaccharides and sialyl oligosaccharide phosphates from bovine colostrum and human urine. Methods Enzymol. 138:289-300.

Saito, T., T. Itoh, and S. Adachi. 1984. Presence of two neutral disaccharides containing $N$-acetylhexosamine in bovine colostrum as free forms. Biochim. Biophys. Acta 801:147-150.

Sánchez-Díaz, A., M. J. Ruano, F. Lorente, and P. Hueso. 1997. A critical analysis of total sialic acid and sialoglycoconjugate contents of bovine milk-based infant formulas. J. Pediatr. Gastroenterol. Nutr. 24:405-441.

Svennerholm, L. 1957. Quantitative estimation of sialic acids: II. A colorimetric resorcinol hydrochloric method. Biochim. Biophys. Acta 24:604-611.

Urashima, T., T. Saito, K. Ohmisya, and K. Shimazaki. 1991. Structural determination of three neutral oligosaccharide in bovine (Holstein-Friesian) colostrum including a novel trisaccharide: GalNAc $\alpha 1-3 \mathrm{Gal} \alpha$ 1-4 Glc. Biochim. Biophys. Acta 1073: 225-229.

Veh, R. W., J. C. Michalski, A. P. Corfield, M. Sander-Vewer, D. Gies, and R. Schauer. 1981. New chromatographic system for the rapid analysis and preparation of colostrum sialyl oligosaccharides. J. Chromatogr. 212:313-322.

Viverge, D., L. Grimmonprez, G. Cassanas, L. Bardet, and M. Solere. 1986. Diurnal variations and within the feed in lactose and oligosaccharides of human milk. Ann. Nutr. Metab. 30:196-209. 